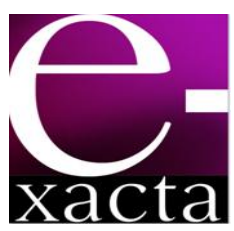

ISSN: 1984-3151

\title{
DETERMINAÇÃo DO TEOR DE GLICEROL LIVRE POR ESPECTROSCOPIA NIR
}

\author{
Determination of Free Glycerol Content by NIR \\ SPECTROSCOPY
}

\section{Giovana de Menezes Rodrigues ${ }^{1}$; Gilmar Roberto Tavares²; Bruna Tais Ferreira de Mello ${ }^{3}$; Camila da Silva ${ }^{4}$}

1 Graduanda em Engenharia de Alimentos. Universidade Estadual de Maringá, 2016. Umuarama, PR. giovanademr@gmail.com.

2 Mestrando em Ciências Agrárias. Universidade Estadual de Maringá, 2015. Engenheiro de Alimentos, Universidade Federal de Santa Catarina. Umuarama, PR. eng.gilmartavares@gmail.com.

3 Mestranda em Bioenergia. Universidade Estadual de Maringá, 2015. Graduada em Química Industrial, Universidade Paranaense. Umuarama, PR. bruna.tf.mello@gmail.com.

4 Doutora em Engenharia Química. Universidade Estadual de Maringá, 2009. Professora do Departamento de Tecnologia. Universidade Estadual de Maringá, Umuarama, PR. camiladasilva.eq@gmail.com.

Recebido em: 01/09/2015 - Aprovado em: 20/11/2015 - Disponibilizado em: 30/11/2015

RESUMO: A análise do teor de glicerol livre (GL) em processos para obtenção do biodiesel é aplicada para avaliar o progresso da reação e etapas de separação, desta forma, neste trabalho é proposto um método rápido e de baixo custo para determinação de GL em biodiesel. Buscando tal propósito foi aplicada a espectroscopia no infravermelho próximo (NIR) associada à quimiometria utilizando misturas de biodiesel etílico de soja, glicerol e etanol no desenvolvimento do método. Foram reportados valores de erro médio quadrático de 0,31 e 0,45 para a calibração e validação, respectivamente. O método foi testado para predição de glicerol livre em amostras obtidas da reação de transesterificação enzimática de óleo de soja e etanol, relatando-se erro médio quadrático de 0,41 e coeficiente de determinação (R2) $>0.98$.

PALAVRAS-CHAVE: Transesterificação. Biodiesel. Método analítico. NIR. Quimiometria.

ABSTRACT: The analysis of free glycerol content (GL) in process for obtaining biodiesel is applied to assess the progress of the reaction and separation steps, thus, this work proposes a fast and inexpensive method for determination of GL in biodiesel. For this purpose it was it was applied to near infrared spectroscopy (NIR) associated with chemometrics using mixtures of ethyl soybean biodiesel, glycerol and ethanol in method development. It has been reported RMSE values of 0.31 and 0.45 for calibration and validation, respectively. The method was tested for free glycerol prediction on samples of the enzymatic transesterification of soybean oil and ethanol, reporting RMSE of 0.41 and coefficient of determination (R2)> 0.98 .

KEYWORDS: Transesterification. Biodiesel. Analytical method. NIR. Chemometrics. 


\section{INTRODUÇÃo}

O biodiesel é uma alternativa para substituir o diesel de petróleo por ser renovável, não tóxico e biodegradável. Na sua queima, há uma menor emissão de gases causadores do efeito estufa, diminuindo os impactos ambientais (ROSA et al., 2014; ANG et al., 2014). O programa nacional de biodiesel (lei $n^{0} 11.097$ de janeiro de 2005) prevê a adição de um percentual mínimo de biodiesel ao óleo diesel comercializado em todo o território nacional, sendo que a partir de novembro de 2014, este percentual é de $7 \%$. Com base nesse cenário, o Brasil figura entre os maiores produtores e consumidores de biodiesel do mundo, com uma produção anual, em 2014, de aproximadamente 3,4 bilhões de litros (ANP, 2015).

A transesterificação de triacilglicerídeos, de origem animal ou vegetal, com um álcool é a metodologia tradicionalmente utilizada para obtenção deste biocombustível, sendo gerado glicerol como subproduto. O glicerol deve ser removido do biodiesel antes da sua comercialização, visto que pode causar danos ao motor e produzir gases tóxicos durante a combustão (KARAOSMANOGLU et al., 1996; HAYYAN et al. 2010). A determinação do teor de glicerol deve ser realizada buscando atender as especificações do produto (MEHER et al., 2006), avaliar a eficiência dos processos de separação aplicados (PEEREBOOM et al., 2007; VASQUES et al., 2013; ROCHA et al., 2014) e monitorar o progresso da reação (MONTEIRO et al., 2008).

A titulometria utilizando periodato de sódio como reagente é um método simples utilizando na determinação do teor de glicerol livre (COCKS; VAN REDE, 1966; PISARELLO et al., 2010), no entanto, a metodologia requer longo tempo de análise e 0 elevado consumo de reagentes e solventes. As metodologias oficiais recomendadas pela Agência Nacional do Petróleo, Gás Natural e Biocombustíveis
- ANP (ABNT - NBR 15908, 2012) e Associação Espanhola de Normativas e Certificações (EN 14105, 2003) indicam a análise utilizando cromatografia em fase gasosa com derivatização prévia da amostra. Tais métodos apresentam alto custo, devido ao consumo de solventes, padrões cromatográficos e derivatizantes, e longo tempo de análise. A utilização da técnica de cromatografia líquida de alta eficiência pode ser utilizada na quantificação de glicerol livre com menor tempo de análise (HÁJEK et al., 2006), no entanto, a técnica exige o elevado consumo de solventes utilizando como fase móvel.

A espectroscopia no infravermelho próximo (NIR) apresenta-se como alternativa aos métodos comumente utilizados, pois se trata de procedimento analítico rápido, reprodutível e com baixo custo por análise quando comparada às técnicas convencionais (HUANG et al., 2008; BAILABIN; SMIRNOV, 2011), visto que pode reduzir ou eliminar o consumo de solvente/reagentes e o tempo de análise. Nos últimos anos, a espectroscopia NIR tem recebido crescente aceitação em processos industriais como opção para o controle de processos industrial (BLANCO et al., 2006). No entanto, a aplicação desta metodologia para determinar o teor de glicerol é pouco explorada na literatura, 0 que despertou interesse no desenvolvimento desta pesquisa.

O presente estudo está inserido em um amplo projeto que visa o estabelecimento de métodos simples, rápidos e de baixo custo, utilizando espectroscopia NIR na determinação de compostos de interesse na produção de biodiesel (RASPE et al., 2011; RASPE; SILVA, 2013; RASPE et al., 2013). Dessa forma, o objetivo do presente trabalho foi aplicar a espectroscopia no infravermelho próximo (NIR) associada à quimiometria para determinação do teor de glicerol livre (GL) em misturas de biodiesel+glicerol+etanol, bem como em amostras obtidas diretamente da reação de óleo de soja e etanol. 


\section{MATERIAIS e MÉTOdOS}

No desenvolvimento do método foram utilizadas amostras de biodiesel etílico de soja, etanol absoluto (Merck) e glicerol (Sigma Aldrich). O biodiesel utilizado foi obtido por transesterificação homogênea alcalina e apresentou $97,5 \%$ de ésteres. A enzima imobilizada de Mucor miehei (Lipozyme IM), cedida pela LNF Latino Americana, óleo de soja (Soya) e etanol absoluto (Merck) foram utilizados na reação de transesterificação enzimática. Para quantificação do teor de glicerol livre utilizou-se periodato de sódio e etileno glicol de procedência Vetec, ácido sulfúrico (Anidrol, 98\%), indicador azul de bromotimol (Synth) e hidróxido de sódio (Anidrol, 97\%).

Amostras preparadas com diferentes percentuais de glicerol, 0 a 10\% (em relação à massa de biodiesel), adicionados ao biodiesel e etanol foram utilizadas nas etapas de calibração e validação, com o objetivo de obter amostras sintéticas condizentes com o meio reacional de transesterificação do óleo de soja. A massa da mistura biodiesel+glicerol e do etanol foram mantidas fixas em 6 e 2,5 g, respectivamente. Foram utilizadas 44 e 22 amostras para a calibração e validação do método, respectivamente.

Os espectros de absorbância na região do infravermelho próximo foram adquiridos, em triplicata, utilizando espectrofotômetro FT-NIR N200 (Büchi) a temperatura ambiente, utilizando placa de vidro $(1,7$ $\mathrm{cm}$ de altura e diâmetro de $9 \mathrm{~cm}$ ) no intervalo espectral de 1000 para $2500 \mathrm{~nm}$. Os tratamentos do espectro, para evitar flutuações da linha de base, foram aplicados e modelos de calibração foram construídos utilizando o software NIRCal 4.01 (Buchi) e regressão por mínimos quadrados parciais (PLS). Os métodos propostos foram avaliados com base no número mínimo de fatores do método PLS e no erro médio quadrático de calibração (RMSEC) e de validação (RMSEV), calculados conforme reportado por Raspe et al. (2013).
O modelo quimiométrico foi validado com novas amostras (validação externa) obtidas a partir da transesterificação enzimática do óleo de soja e etanol e em diferentes condições reacionais, originando amostras com diferentes teores de glicerol. As reações foram conduzidas com base no trabalho de Oliveira et al. (2005), utilizando $20 \%$ de enzima, temperatura de $35 \stackrel{\circ}{\circ}$, razão molar óleo:etanol de 1:3 nos tempos de 2 a 24 horas. As amostras, 8 no total, foram obtidas do meio reacional e enviadas diretamente para análise, em triplicata. Com os resultados obtidos determinou-se o erro médio quadrático de predição (RMSEP) e a correlação linear entre os métodos utilizando o software Statistica® 7.0 (Statsoft Inc).

Os percentuais de glicerol, das etapas de calibração, validação e predição, foram determinados utilizando como referência o método de periodato de sódio (COCKS; VAN REDE, 1966), o qual consiste na medida do iodato formado pela reação do periodato de sódio com glicerol (FILHO; MICKE, 2007).

\section{Resultados e Discussão}

A FIG. 1 apresenta o espectro característico da mistura biodiesel etílico de soja+etanol+glicerol obtido na região do infravermelho próximo, o qual é semelhante ao apresentado por Blanco et al. (2006). A região entre 2000 a 2200 nm corresponde à região de absorção do glicerol, devido às bandas de combinação da ligação O-H (BLANCO et al., 2006; FONTALVO-GÓMEZ et al., 2013). O espectro obtido apresentou variabilidades em 1700 e 2300 nm, Pinzi et al. (2012) apresentam que estes comprimentos de onda correspondem à absorção dos ésteres de ácidos graxos.

Os dados espectrais foram tratados por diferentes métodos, sendo os resultados obtidos apresentados na TAB. 1. Os menores valores para o RMSEC e 
RMSEV $(0,31$ e 0,45$)$ foram obtidos para o prétratamento por $1^{\text {a }}$ derivada, com 9 pontos, pelo método de Savitzky-Golay com correção da linha de base e suavização. Blanco et al. (2004) reportam a aplicação de espectroscopia NIR para avaliar a esterificação de glicerol e ácidos graxos livres e relatam RMSEC de 1,0 para a etapa de calibração da metodologia, utilizando dados na região de 1100 a $2500 \mathrm{~nm}$ com pré-tratamento por $1^{\text {a }} \stackrel{\text { derivada. }}{ }$

Os valores de RMSE conforme o número de fatores utilizados no método PLS são reportados na FIG. 2, sendo possível constatar que o erro diminui com o aumento do número de fatores utilizados.

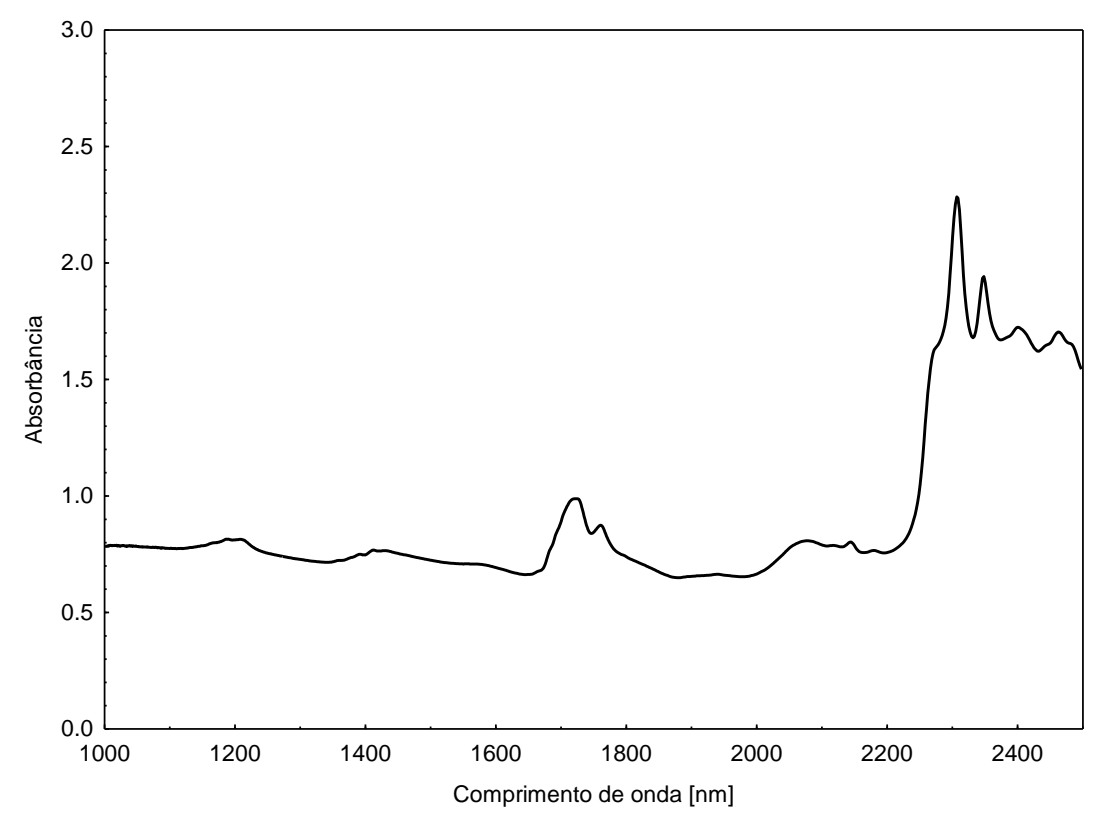

Figura 1 - Espectro de absorbância da mistura biodiesel etílico de soja+etanol+glicerol.

Tabela 1

Resultados de diferentes métodos de pré-tratamento dos dados, obtidos entre 1000 a $2500 \mathrm{~nm}$, no desenvolvimento do método para teor de GL (\%)

\begin{tabular}{|c|c|c|c|c|}
\hline & \multicolumn{4}{|c|}{ Parâmetro } \\
\hline & $\mathbf{R}^{2}$ calibração & $\mathbf{R}^{2}$ validação & RMSEC & RMSEV \\
\hline Correção da Linha de Base ${ }^{a}$ & 0,9928 & 0,9819 & 0,68 & 0,54 \\
\hline${ }^{\mathrm{a}}+$ Suavização SG ${ }^{\mathrm{b}}$ & 0,9932 & 0,9791 & 0,36 & 0,58 \\
\hline${ }^{a, b}+1^{a}$ Derivada $^{c}$ & 0,9952 & 0,9887 & 0,31 & 0,45 \\
\hline${ }^{\mathrm{a}, \mathrm{b}}+2^{\mathrm{a}}$ Derivada $^{\mathrm{a}}$ & 0,9924 & 0,9701 & 0,38 & 0,60 \\
\hline
\end{tabular}




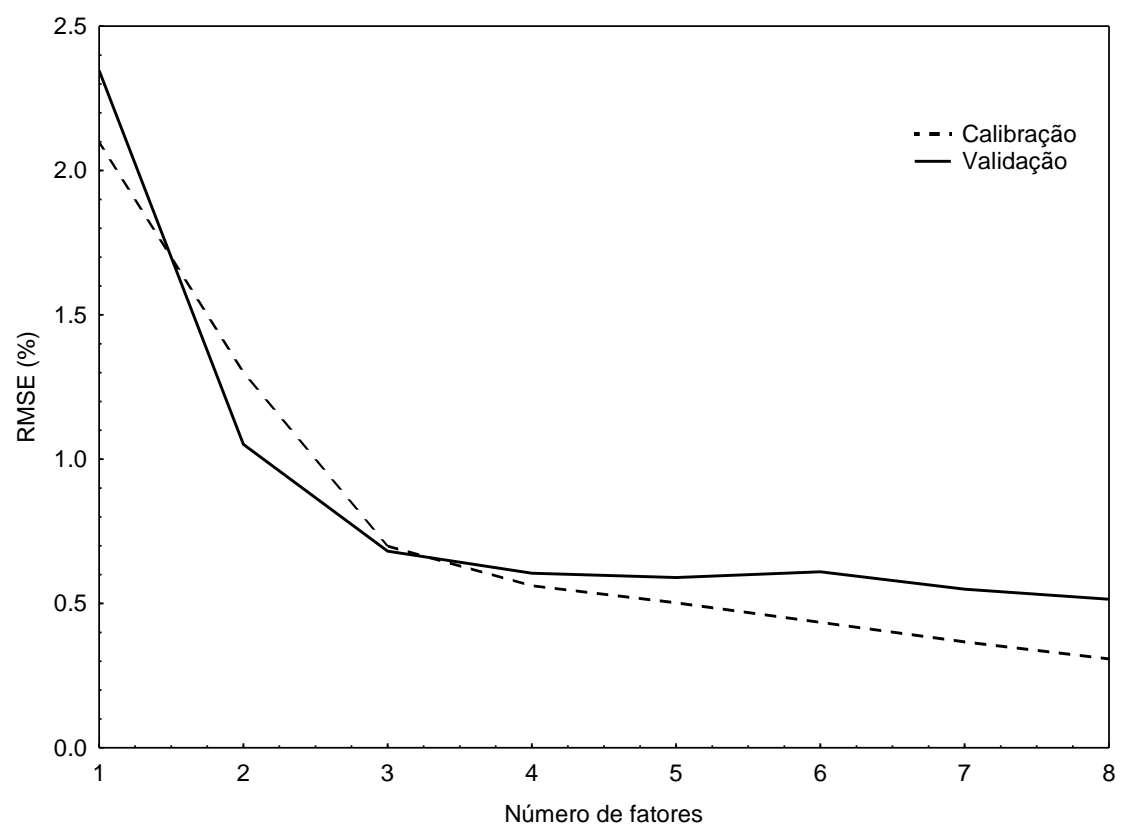

Figura 2 - Valores de RMSE em função do número de fatores utilizados no desenvolvimento do método.

A partir dos dados apresentados na TAB. 1 constatase que 0 modelo de calibração desenvolvido apresentou bom desempenho na determinação do teor de GL, com alta correlação entre os valores observados e preditos $\left(R^{2}>0,99\right)$. O método NIR/PLS desenvolvido foi testado para determinação do teor de
GL em amostras obtidas da transesterificação enzimática do óleo de soja e etanol (sem nenhum tratamento prévio). A relação linear entre os métodos é apresentada na FIG. 3, sendo obtido RMSEP de 0,41 e coeficiente de determinação $\left(R^{2}\right)>0,98$.

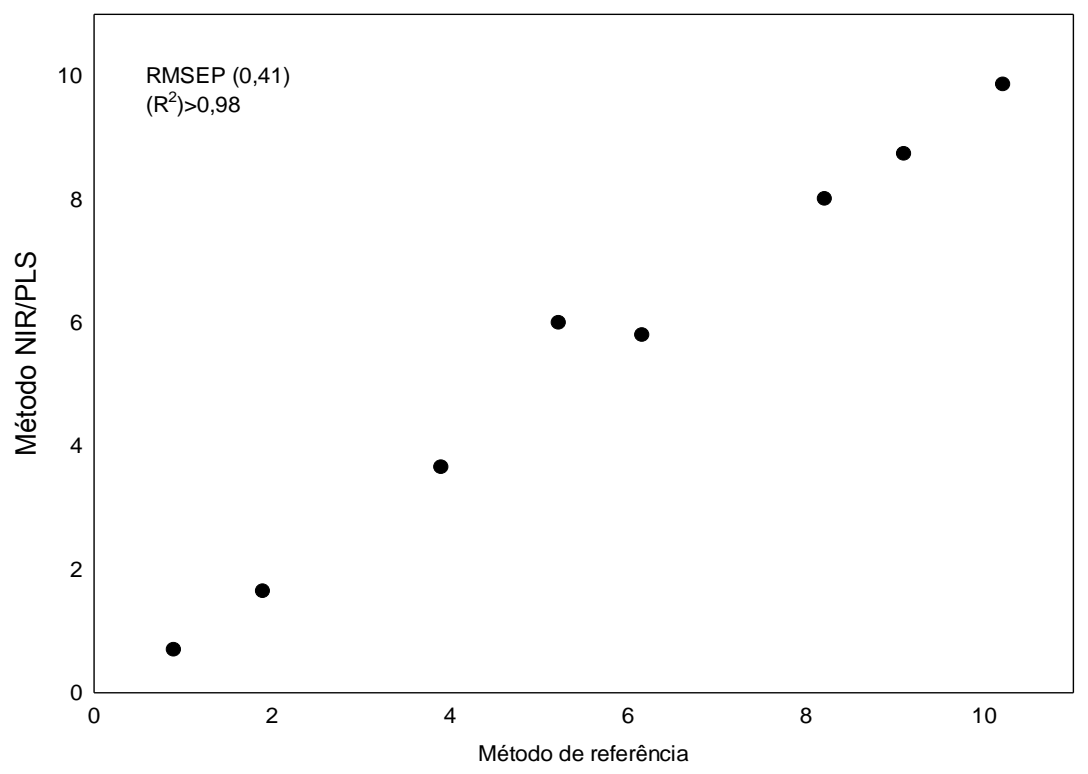

Figura 3 - Correlação entre o teor de GL predito pelo método NIR/PLS e observado pelo método de referência. 
A avaliação do teor de glicerol em biodiesel utilizando espectroscopia UV-VIS e NIR é relatada no trabalho de Dorado et al. (2011). Os autores obtiveram correlação de 0,75 e 0,81 para os métodos utilizando UV-VIS+NIR e UV-VIS, respectivamente. No trabalho de Pinzi et al. (2012) foi proposto o monitoramento da transesterificação metílica utilizando espectroscopia no infravermelho próximo e relata-se correlação de 0,65 entre os dados preditos pelo NIR e observados pelo método EN 14105 (2003) para o teor de glicerol livre. O estudo de Beneti et al. (2013) relata a eficiência da espectroscopia NIR para quantificação de glicerol na determinação do equilíbrio líquidolíquido de misturas de ésteres+água+etanol+glicerol.

Os resultados obtidos neste estudo podem ser comparados com outras técnicas propostas na literatura. Pisarello et al. (2010) apresentam erros de 2 a $8 \%$ na determinação do teor de GL utilizando método de periodato de sódio. Valdez et al. (2012) propuseram a determinação de GL em amostras de biodiesel por método enzimático com detecção colorimétrica obtendo correlação de 0,98 entre 0 método proposto e método oficial EN 14105 (2003). No entanto, cabe destacar que se trata de método que requer a extração do glicerol e uso de reagentes químicos. O método desenvolvido por Filho e Micke (2007) para determinação de GL em biodiesel por eletroforese capilar apresenta erros de 2 a 4,6\%.

\section{Conclusão}

A determinação do teor de glicerol livre por espectroscopia NIR e ferramentas quimiométricas foi proposta neste trabalho. Os resultados obtidos relatam valores de RMSE de 0,31, 0,45 e 0,41 para as etapas de calibração, validação e predição, respectivamente. O método desenvolvido e a metodologia de referência apresentam alta correlação $\left(R^{2}>0,98\right)$. Com base nos resultados do presente estudo o método NIR/PLS desenvolvido pode ser utilizado na determinação do glicerol em processos da cadeia produtiva do biodiesel.

\section{AGRADECIMENTOS}

Os autores agradecem à LNF Latino Americana pela doação das enzimas e ao CNPq pelo suporte financeiro.

\section{REFERÊNCIAS}

ABNT - NBR 15908: Biodiesel — Determinação da glicerina livre, monoglicerídeos, diglicerídeos, triglicerídeos e glicerina total por cromatografia gasosa, Associação Brasileira de Normas Técnicas, 2012.

ANG, G. T. et al. Recent development and economic analysis of glycerol-free processes via supercritical fluid transesterification for biodiesel production.

Renewable and Sustainable Energy Reviews, v. 31, 61-70, 2014.

AGÊNCIA NACIONAL DO PETRÓLEO, GÁS NATURAL E BIOCOMBUSTÍVEIS - ANP. Resolução ANP No 14, de 11.5.2012 - DOU 18.5.2012. Disponível em:

http://nxt.anp.gov.br/nxt/gateway.dll/leg/resolucoes_an p/2012/maio/ranp\%2014\%20-\%202012.xml. Acesso em: abr. 2015.

BALABIN, R. M.; SMIRNOW, S. V. Variable selection in near-infrared spectroscopy: Benchmarking of feature selection methods on biodiesel data. Analytica Chimica Acta, v. 602, 63-72, 2011.

BENETI, S. C. et al. Determination of liquid-liquid equilibrium data for biodiesel containing ternary systems using near infrared technique. Brazilian Journal of Chemical Engineering, v. 30, 23-31, 2013.

BLANCO, M. et al., Analytical control of an esterification batch reaction between glycerine and 
fatty acids by near-infrared spectroscopy. Analytical Chimica Acta, 51, 143-148, 2004.

BLANCO, M. et al., Application of multivariate curve resolution to chemical process control of an esterification reaction monitored by near-infrared spectroscopy. Applied Spectroscopy, v.60, 641-647, 2006

COCKS, L. V.; VAN REDE, C. Laboratory handbook for oils and fats analysis. London: Academic Press, 1966.

DORADO, M. P. et al. Visible and NIR spectroscopy to assess biodiesel quality: Determination of alcohol and glycerol traces. Fuel, v. 90, 2321-2325, 2011.

EN 14105: Determination of free and total glycerol and mono-, di-, triglyceride contents, issued by Asociación Española de Normalización y Certificación, Madrid, 2003.

FILHO, L.C.G.; MICKE, G.A. Development and validation of a fast method for determination of free glycerol in biodiesel by capillary electrophoresis. Journal of Chromatography A, v.1154, 477-480, 2007.

FONTALVO-GÓMEZ, M. et al. In-line Near-infrared (NIR) and Raman spectroscopy coupled with principal component analysis (PCA) for nn situ evaluation of the transesterification reaction. Applied Spectroscopy, v. 67, 1142-1149, 2013.

HÁJEK, M. et al. Determination of free glycerol in biodiesel. European Journal of Lipid Science and Technology, v. 108, 666-669, 2006.

HAYYAN, M. et al. A novel technique for separating glycerine from palm oil-based biodiesel using ionic liquids. Fuel Processing Technology, v. 91, 116120, 2010.

HUANG, H. et al. Near infrared spectroscopy for on/inline monitoring of quality in foods and beverages: $A$ review. Journal of Food Engineering, v. 87, 303-313, 2008.

KARAOSMANOGLU F. et al. Investigation of the refining step of biodiesel production. Energy \& Fuels, v. 10, 890-895, 1996.

MEHER, L. C. et al. Technical aspects of biodiesel production by transesterification - a review.

Renewable and Sustainable Energy Reviews, v. 10, 248-268, 2006.
MONTEIRO, M. R. et al. Critical review on analytical methods for biodiesel characterization. Talanta, v. 77, 593-605, 2008.

OLIVEIRA, D. et al. Kinetics of Enzyme-Catalyzed Alcoholysis of Soybean Oil in $n$-Hexane. Applied Biochemistry and Biotechnology, v. 231, 121-124, 2005.

PINZI, S. et al. Near infrared reflectance spectroscopy and multivariate analisys to monitor reaction products during biodiesel production. Fuel, v. 92, 354-359, 2012.

PISARELLO, M. L. et al. Volumetric method for free and total glycerin determination in biodiesel. Industrial and Engineering Chemistry Research, v. 49, 89358941, 2010.

RASPE, D.T. et al., Determinação de Ácidos Graxos Livres em Óleos Vegetais por FT-NIR e Calibração Multivariada. Revista Ciências Exatas e Naturais, v.13, 1-10, 2011.

RASPE, D. T.; SILVA, C. Determination of Free Fatty Acid by FT-NIR Spectroscopy in Esterification Reaction for Biodiesel Production. Journal of Energy, v. 2013, 1-5, 2013.

RASPE, D. T. et al. Determinação de Ésteres Etílicos de Ácidos Graxos por Espectroscopia FT-NIR e Calibração Multivariada em Reações de Transesterificação. E-xacta, v. 6, 27-33, 2013.

ROCHA, E. G. A. et al. Liquid-liquid equilibria for ternary systems containing ethylic palm oil biodiesel + ethanol + glycerol/water: Experimental data at 298,15 and $323,15 \mathrm{~K}$ and thermodynamic modeling. Fuel, $\mathrm{v}$. 128, 356-365, 2014.

ROSA, H. A. et al. Biodiesel produced from crambe oil in Brazil A study of performance and emissions in a diesel cycle engine generator. Renewable and Sustainable Energy Reviews, v. 38, 651-655, 2014.

VALDEZ, H. C. et al. Determinacão de glicerol livre e total em amostras de biodiesel por método enzimático com detecção colorimétrica. Química Nova, v. 35, n. 3, 601-607, 2012.

VASQUES, E. C. et al. Adsorption of glycerol, monoglycerides and diglycerides present in biodiesel produced from soybean oil. Environmental Technology, v. 34, 2361-2369, 2013. 